\title{
Impact of patient-perceived quality of primary health care on the patients' bypass behaviour in China
}

\section{WeiWei Zhang}

The Research center of National Drug Policy \& Ecosystem, China Pharmaceutical University

\section{Yuankai Huang}

The Research Center of National Drug Policy \& Ecosystem, China Pharmaceutical University

\section{Gaojie Li}

The Research Center of National Drug Policy \& Ecosystem, China Pharmaceutical University

Hongjie Zhou

The Research Center of National Drug Policy \& Ecosystem, China Pharmaceutical University

Xiaoyu Xi ( $\nabla$ xixy@cpu.edu.cn )

The Research Center of National Drug Policy \& Ecosystem, China Pharmaceutical University

\section{Research article}

Keywords: bypass behaviour, primary health care, perceived quality, China

Posted Date: March 23rd, 2020

DOI: https://doi.org/10.21203/rs.2.21413/v2

License: (c) (i) This work is licensed under a Creative Commons Attribution 4.0 International License. Read Full License 


\section{Abstract}

Background: Bypass behaviour is a phenomenon that an individual visits an higher-level and farther health institution instead of one with an acceptable quality of care that is closer. Patient bypass often occurs under the loose rules of first diagnosis. Additionally, patients' perceived quality of primary health care is one of the important factors affecting their bypass behaviours. Objective: to explore individuals' bypass behaviour in China and the impact of patients' perceived quality of primary health care on their bypass behaviour.

Method: in 2019, a national wide sampling survey was conducted to collect data of Chinese residents' bypass behaviours and its potential influencing factors. The questionnaire that was used assessed the following: the perceived quality of primary care using the Primary Care Assessment Tool; bypass experience; age; health insurance; and other factors. A logit regression model was used to analyse the impact of perceived quality of $\mathrm{PHC}$ on bypass behaviour.

Results: the data of 2070 residents sampled in 706 secondary/tertiary hospitals in China were collected. The results show that perceived quality of PHC is significantly associated with bypass behaviour in China. Per increase of one the PCAT mean score had an odds ratio of $0.98(\mathrm{p}<0.05)$ for bypass behaviour. Gender, household registration and cold fee for $\mathrm{PHC}$ were negatively associated with bypass behaviour.

Conclusion: patients' perceived quality of primary health care plays an active role in reducing their bypass behaviours, which may provide a new perspective for the design of primary health care policies.

\section{Introduction}

Primary health care (PHC), as the core of the health system, is provided by primary health care institutions (PHCls). Each PHClprovides comprehensive, coordinated and integrated health care to individuals, families, and communities within its service areas(1). PHCls is construct an effective network to expand the coverage of health services in countries with limited health resources(2). In countries with a national hierarchical health care system (NHMS), PHC can address the common diseases of residents at a lower cost and improve their welfare, which is cost effective. Therefore, most countries around the world have invested massive resources to build $\mathrm{PHCls}$ and strengthen $\mathrm{PHC}$ service delivery. As an NHMS country, the Chinese health care system is a 3-level hierarchical network. PHCls serve as "gatekeepers" to take care of patients with common and frequently occurring diseases; secondary hospitals are regional hospitals that provide comprehensive health care to multiple communities and undertake certain teaching and scientific research tasks; and tertiary hospitals, which are usually composed of provincial and municipal general hospitals, teaching hospitals, and specialized hospitals, implement teaching and scientific research tasks and conduct the diagnosis and treatment of patients with difficult, miscellaneous and critical diseases(3).

However, it does not seem easy to gain benefits of investment in PHC. Due to the lack of funds and equipment and the inadequate training of medical staff during an early stage in development of primary 
health care systems, many countries (such as India, Saudi Arabia, and China) have imperfect PHC systems, and the quantity and quality of health resources in PHCls are generally lower than those in higher-level hospitals (4). In addition to the asymmetry of medical information, patients have limited choice besides bypassing the PHCls and go to higher-level hospitals for better health care.

Bypass is generally defined as the behaviour of patients visiting an higher-level health institution instead of one with an acceptable quality of care and closer to them, which reflects the patients' preference for better health care(5-9). In developing countries where the PHC system is imperfect, it is more common for patients to bypass their nearest PHCls for higher-level health care institutions. With the development of the primary health care system, this conventional concept and behaviour, which is often referred to as "bypass behaviour", is still preserved(5). For example, the data show that the total number of medical visits in various health institutions in China in 2017 was 8183.11 million person-times, while the PHCls accounted for only 4428.92 million person-times (54.12\%), which indicates that patients bypass the PHCls to a large extent(10).

In developing countries such as China, where the PHC system is imperfect, it is more common for patients to bypass their nearest primary care facility and go to higher-level health care institutions. The prevalence of patients' bypass has negative impacts on the health system, the health care institutions and the individuals. For the health system, bypass behaviour hinders the operational efficiency of the health system(11), which is not conducive to the overall operation and development of health system and affects the government's strategy on the input of PHC resources. For health care institutions, bypass behaviour limits the utilization of services provided by PHCls to a certain extent, which reduces the employment of PHC providers, and may even lead to the closure of PHCls(12). Meanwhile, bypass behaviour will increase visits to higher-level hospitals, resulting in a decrease in the efficiency of the health system. For individuals, bypassing the nearest $\mathrm{PHCls}$ to seek further treatment will cost more time and money, which may hardly be cost-effective. Therefore, reducing bypass behaviour, which is of practical significance, contributes to the greater well-being of the health system and individuals.

Many studies have tried to explain the cause of bypass behaviour to identify the paths of reducing these behaviours. Studies have shown that socioeconomic conditions (such as income), demographic factors (such as gender), the health status of patients (such as disease severity) $(13,14)$, geographic factors, medical expenses(9), and the quality of health care institutions(15) can affect patients' bypass behaviour. Among these factors, the quality of health care institutions is the fundamental cause of patients' bypass behaviour. Rao, K. D., et al. (2018)(13) explored the effects of the structural quality of health centres on the bypass behaviour of Indian patients, and the results suggest that improvements in structural quality have a positive impact on the reduction in bypass behaviour. Akin et al. (1999) (9) studied the bypass behaviour of Sri Lankan residents and the characteristics of bypassed health care institutions. The results show that the quality and price of health services are important factors influencing bypass behaviour. Leonard et al (2003) (11), Kruk et al (2009) (16), and Gauthier et al (2011) (8) studied the bypass behaviour and its influencing factors among African residents. The focus of these studies is on the quality of health care services. Among these studies, Gauthier analysed the influence of 
subjective quality perception and objective quality on bypass behaviour, but the measurement indicators of subjective quality perception are controversial. Aoki, T. et al (2018) (5) studied the influence of subjective quality perception (medical experience indicators) on the bypass behaviour of Japanese residents. The results indicated that the better patients' experience of primary care institutions is, the fewer times they bypass gatekeepers. It is obvious that the objective quality of the health caremedical institution and patients' subjective perception of the quality of PHC services may have varying degrees of impact on their bypass behaviour.

Based on existing studies, perceived quality of health services affects residents' bypass behaviour. A literature review indicates that subjective quality perception can explain patients' bypass behaviour on a broader level, which is irreplaceable by the objective quality of PHCls. For example, practical experience has shown that patients sometimes bypass PHCls (perceived lower quality) and instead seek health care in higher-level health care institutions (perceived higher quality) whose actual advantages in quality of services usually do not add extra health outcomes in the treatment of common or chronic diseases (11). This phenomenon can be explained by the patient's subjective quality perception-to a large extent, the patient's bypass decision depends on his or her own perceived quality of the care delivered by the health care institution instead of the true structural quality of the health caremedical institution.

However, current research on the impact of patients' subjective quality perception on bypass behaviour is limited. To our knowledge, only Japan has conducted similar research(5), and there is no such systematic study in developing countries. At present, China has conducted some studies related to patient bypass behaviour(17-21). However, on the one hand, these studies do not rigorously define bypass behaviour. On the other hand, the selection of quality measurement indicators for health care institutions is often incomprehensive and mostly limited to objective conditions, such as the number of beds in health care institutions(22-24). The changes in the medical model and in health services require the expansion of the connotation of the quality of health services, including comprehensive factors such as work efficiency, cost, service attitude, service availability and service fairness. Therefore, the above studies are not a good representation of the actual quality of health care services and patients' medical experiences. In addition, due to differences in health systems and socio-cultural factors, the results of other countries' research cannot be directly applied to China. Therefore, this study conducted an in-depth exploration of this issue in China, a country that is representative of developing countries.

In summary, this study aims to explore how Chinese patients' perceived quality of PHCls affects their bypass behaviour. Specifically, this study focuses on whether a patient's perceived quality of PHC affects his or her bypass behaviour and the degree and direction of this effect. At present, most of China's policies on primary health care systems are aimed at improving basic health care service hardware, management systems and professional configurations, but the government has not taken action against the problem of low-quality perceptions of PHCls by the residents in China. There are evidences in China that the PHC network have been notably developed and improved in both quality and quantity, but many residents in China are still holding the prejudice that $\mathrm{PHCs}$ are providing low quality health care, and this are hindering the further development of PHC in China(25). If it is proven that improvement in the 
perception of health care service quality can effectively curb the bypass behaviour of Chinese patients, this information would serve as an important reference for the development of China's PHC system. The results and conclusions of this study will serve as a reference for the construction of primary health care services and the improvement of PHC utilization in China and other developing countries.

\section{Materials And Methods}

\section{Participants}

For extended sample coverage reflecting the overall bypassing status of residents in China, this study adopts a multi-stage sampling strategy to extract samples. The steps are as follows: (1) a total of 31 provinces (autonomous regions)/municipalities in mainland China were included, and in each province (autonomous region)/municipality, all cities were divided into three urban groups according to their GDP per capita in 2018, with a total of 93 urban groups included; (2) based on the hospital administrators' willingness, convenience sampling was adopted to select specific hospitals, and at least 2 secondary hospitals and 2 tertiary hospitals were selected in each urban group; and (3) in each hospital, at least 2 outpatients and 2 inpatients were selected according to whether they agreed to participate and met the following criteria.

Respondents participating in the study were required to meet the following conditions: (1) the patient was at least 18 years of age; (2) in the past 12 month the patient visited health care institutions for non-severe health problems (emergency and surgical patients were excluded); (3) the patient was not referred from a $\mathrm{PHCl}$; and (4) respondents had experiences of receiving health care in both primary care institutions and secondary /tertiary hospitals in the past 12 months (5) respondents who indicated that a $\mathrm{PHCl}$ was closest to their home were included in our study. Each eligible respondent was provided with informed consent. To ensure the confidentiality of the information provided by respondents, the researchers began their investigation after obtaining the consent of the respondent.

\section{Dependent variables}

This study used the method of Gauthier (2011) (8) to measure the respondents' bypass behaviour based on the patients' knowledge and judgement of available health caremedical institutions. These respondents were then asked if they had bypassed their nearest PHCls in the past 12 months and went to secondary and tertiary hospitals. If the answer was "yes", it was defined as bypass behaviour, which was to be assigned a value " 1 " and " 0 " otherwise. The method has been proven to be more complete and accurate. Patients who suffered from serious diseases and who were referred to secondary and tertiary hospitals from PHCls were not defined as bypassers.

\section{Independent variables}


This study uses the patients' medical experiences to reflect their perception of the quality of PHC services, and this method is increasingly used to assess the quality of primary and advanced care(5). The Primary Care Assessment Tool (PCAT) instruments developed by the Johns Hopkins Primary Care Policy Center for Underserved Populations were adopted to measure patients' perception of PHC services(26). The PCAT has been used and validated in multiple countries and is perhaps one of the most widely studied and applied tools for measuring the quality of primary care across the globe.

The study used the adapted Primary Care Assessment Tool-Chinese version (PCAT-C) (27). The answer is based on a 4-point Likert scale (definitely $=5$ points; most likely, $=3$ points; possibly $=2$ points; definitely not $=1$ point; not sure/do not know = 9). In keeping with the methods used in PCAT studies in other countries, this study assigns a value of 2.5 for 'not sure/do not remember' answers and uses the average score for the missing data(28). The responses are multiplied by 25 . The PCAT scales representing six primary care domains: First contact $\square$ Continuity $\llbracket$ Coordination $\square$ Comprehensiveness $\square$ Family centeredness $\square$ Community orientation(26). The total score is the mean of the six domain scores and reflects an overall measure of patients' experiences of primary care attributes. Thus, the domain scores range from 0 to 100 points, with higher scores indicating better perception of primary health care.

\section{Covariates}

Based on past empirical studies and literature, this study included covariates to control for other factors that may influence patient bypass behaviour. The study included demographic characteristics, disease characteristics, and health care institution characteristics as control covariates, such as gender, age, household registration, education, annual income, occupation, marital status, number of kids, selfperceived health, basic medical insurance, the cost to $\mathrm{PHC}$, commercial medical insurance and the numbers of, the average distances to, the average time of transportation to, and the average waiting time in $\mathrm{PHCls}$ and nearest tertiary \secondary hospitals, which have been proven in previous literature to affect patients' bypass behaviour( $5,8,11,12,15)$. Among these covariates, basic medical insurance is divided into Urban Employee Basic Medical Insurance (UEBMI), Urban Resident Basic Medical Insurance (URBMI) and New Cooperative Medical Scheme (NCMS). The classification criteria for other variables are detailed in the results section. From the perspective of patients, the facility information in the enriched dataset, such as the charges for health care, is exogenous. That is, each individual is linked to the characteristics of available services, not the services that were actually used. Thus, the endogeneity problem that arises when this information is collected from respondents is avoided.

\section{Pre-test of questionnaire}

Data from a total of 106 patients were collected in the secondary and tertiary hospitals of Hangzhou, Zhejiang Province, China, according to their willingness to participate in the survey by convenience sampling. The study verified the rationality, comprehensibility and readability of the entire questionnaire and tested the reliability and validity of the PCAT scale. The results showed that the questionnaire was 
reasonable, understandable and readable. All indicators (standardized Cronbach's alpha $=0.688$, KaiserMeyer-Olkin measure of sampling adequacy $=0.673$, significance of Bartlett's test of sphericity $=0.000$ ) indicated acceptable reliability and validity for the PCAT in measuring perceived quality of PHC of the population of this study.

\section{Data collection}

We organized and conducted this survey nationwide. Postgraduate students majoring in public health were recruited as candidate data auditors, and undergraduates majoring in public health were recruited as candidate data collectors. The researchers provided training and testing of survey skills for all of them, and 10 data auditors and 500 data collectors passed the test and were formally recruited. The data collectors conducted the survey in sampled secondary and tertiary hospitals, and the data auditors randomly checked $5 \%$ of the collected questionnaires at the end of the survey. The data were entered by two different persons in a double-blind method.

All of the research data were collected by team-designed software (for Android: http://117.78.41.192:8088/bistone/framework/app/mianFangMaster.apk) and for iOS: https://itunes.apple.com/us/app/\%E9\%9D\%A2\%E8\%AE\%BF\%E5\%A4\%A7\%E5\%B8\%88/id1063924434? $\mathrm{mt}=8$ ) and were processed into identifiable electronic data.

\section{Statistical analysis}

This study used descriptive statistics to report whether respondents had bypass behaviour (with yes=1, no $=0$ ) in the past year, as well as patients' scores in six core domains of the PCAT scale: first contact, longitudinal/ongoing care, coordination, comprehensiveness (services provided), family-centeredness and community orientation. In addition, we report the means and standard deviations of the continuous data for the covariates and the frequencies and percentages for the categorical data. Student's t-tests were used to analyse continuous data, and chi-square tests were used to analyse the categorical data. Because bypass measurements are binary categorical variables, this study adopted logit regression models to analyse the effect of patient PCAT scores on bypass behaviour. The means and Bayesian $95 \%$ confidence intervals (Cls) were estimated and expressed as odds ratios (ORs) for presentation. We used VIF test to test the multicollinearity of the independent variables, and the multicollinearity was acceptable. All statistical analyses were performed in SPSS 24 and STATA 14 software.

\section{Results}

Table 1 presents the descriptive statistics of the final sample. The total number of questionnaires collected from 31 provinces was 2070, and the effective response rate of the questionnaire was $67.44 \%$. The data involved 706 hospitals of different levels, $52.85 \%$ of 
which were secondary hospitals and $46.47 \%$ of which were tertiary hospitals. In the valid questionnaire, the mean age of the respondents was 38.97 years, and the ratio of male to female respondents was approximately 1:1.60. This study included participants in both urban and rural areas, with a ratio of rural to urban respondents of approximately 1:1.52. Most of the urban respondents were insured by the UEBMI or the URBMI, most rural respondents were insured by the NCMS (30.16\%); only a small proportion of the respondents (3.80\%) reported that they did not have any form of health insurance. The results also showed that more than half of the respondents $(75.93 \%)$ indicated that they engaged in bypass behaviour in the past year (1-5 times for most respondents).

Table 1. Characteristics of Patients With or Without Bypass Behaviour $(\mathrm{N}=1396)$ 


\begin{tabular}{|c|c|c|}
\hline Variable & Frequency/Mean & Proportion/SD \\
\hline Age & 38.97 & 16.88 \\
\hline PCAT scores (mean and SD of total score) & 67.26 & 6.90 \\
\hline First contact & 66.67 & 9.92 \\
\hline Continuity & 70.01 & 8.42 \\
\hline Coordination & 70.356 & 13.61 \\
\hline Comprehensiveness & 67.62 & 12.81 \\
\hline Family centeredness & 70.80 & 15.86 \\
\hline Community orientation & 58.13 & 17.02 \\
\hline \multicolumn{3}{|l|}{ Bypass behaviour } \\
\hline No & 313.00 & $22.42 \%$ \\
\hline Yes & 1060.00 & $75.93 \%$ \\
\hline Data missing & 23.00 & $1.65 \%$ \\
\hline \multicolumn{3}{|l|}{ Number of annual bypass behaviours } \\
\hline 0 & 313.00 & $22.42 \%$ \\
\hline $1-5$ & 996.00 & $71.35 \%$ \\
\hline $6-10$ & 46.00 & $3.30 \%$ \\
\hline $11-15$ & 13.00 & $0.93 \%$ \\
\hline $16-20$ & 1.00 & $0.07 \%$ \\
\hline$>20$ & 4.00 & $0.29 \%$ \\
\hline Data missing & 23.00 & $1.65 \%$ \\
\hline \multicolumn{3}{|l|}{ Gender } \\
\hline Male & 536.00 & $38.40 \%$ \\
\hline Female & 860.00 & $61.60 \%$ \\
\hline \multicolumn{3}{|l|}{ Household registration } \\
\hline Rural & 553.00 & $39.61 \%$ \\
\hline Urban & 843.00 & $60.39 \%$ \\
\hline \multicolumn{3}{|l|}{ Medical treatment type } \\
\hline Outpatient & 878.00 & $62.89 \%$ \\
\hline Hospitalization & 518.00 & $37.11 \%$ \\
\hline \multicolumn{3}{|l|}{ Marriage } \\
\hline Single & 467.00 & $33.45 \%$ \\
\hline Married & 897.00 & $64.26 \%$ \\
\hline Other (divorce or separation) & 32.00 & $2.29 \%$ \\
\hline \multicolumn{3}{|l|}{ Number of kids } \\
\hline 0 & 520.00 & $37.25 \%$ \\
\hline $1-3$ & 831.00 & $59.53 \%$ \\
\hline$>3$ & 45.00 & $3.22 \%$ \\
\hline \multicolumn{3}{|l|}{ Education } \\
\hline Elementary school or lower & 149.00 & $10.67 \%$ \\
\hline Junior high school & 208.00 & $14.90 \%$ \\
\hline High school & 282.00 & $20.20 \%$ \\
\hline Junior college & 223.00 & $15.97 \%$ \\
\hline Undergraduate & 498.00 & $35.67 \%$ \\
\hline Graduate student or higher & 36.00 & $2.58 \%$ \\
\hline \multicolumn{3}{|l|}{ Income $\triangle \mathrm{CNY} \square$} \\
\hline$<5529$ & 561.00 & $40.19 \%$ \\
\hline 5529-12,899 & 229.00 & $16.40 \%$ \\
\hline $12,899-20,924$ & 127.00 & $9.10 \%$ \\
\hline 20,924-31,990 & 101.00 & $7.23 \%$ \\
\hline $31,990-59,259$ & 184.00 & $13.18 \%$ \\
\hline
\end{tabular}




\begin{tabular}{lll}
$>59,259$ & 194.00 & $13.90 \%$ \\
Occupation & 272.00 & $19.48 \%$ \\
Student & 100.00 & $7.16 \%$ \\
Teacher & 59.00 & $4.23 \%$ \\
Public servant & 250.00 & $17.91 \%$ \\
Worker & 213.00 & $15.26 \%$ \\
Individual household & 142.00 & $10.17 \%$ \\
Farmer & 140.00 & $10.03 \%$ \\
Retired & 16.00 & $1.15 \%$ \\
Temporary unemployment & 35.00 & $2.51 \%$ \\
Long-term unemployment & 25.00 & $1.79 \%$ \\
Medical staff & 144.00 & $10.32 \%$ \\
Others & & \\
Insurance & 53.00 & $3.80 \%$ \\
None & 405.00 & $29.01 \%$ \\
UEBMI & 421.00 & $30.16 \%$ \\
URBMI & 421.00 & $30.16 \%$ \\
NCMS & 66.00 & $6.87 \%$ \\
Other & & \\
CMI & 958.00 & $68.62 \%$ \\
No & 438.00 & $31.38 \%$ \\
Yes & & \\
Self-health grade & 27.00 & $1.93 \%$ \\
Very poor & 151.00 & $10.82 \%$ \\
Poor & 627.00 & $44.91 \%$ \\
Fair & 432.00 & $30.95 \%$ \\
Good & 159.00 & $11.39 \%$ \\
Very good & & \\
Chronic disease & 1049.00 & $75.14 \%$ \\
No & 347.00 & $24.86 \%$ \\
Yes & & \\
\hline
\end{tabular}

Table 2 shows the health care institutions in the vicinity of the respondents' residence. The results indicated that there were 2 hospitals around most respondents' residence. Only a small number of respondents indicated that there were no hospitals near their residences. Respondents spent an average of 10.57 CNY (Chinese yuan) on PHCI transportation expenses. Meanwhile, approximately $43.05 \%$ of respondents reported that a PHCI was closest to their residence, and $39.68 \%$ of respondents indicated that secondary and tertiary hospitals were closest to their residence. In addition, the distance and time travelled by the respondents to the PHCI and the waiting time at the PHCI were lower than those of the secondary and tertiary hospitals (see Table 2). 
Table 2. Patient-reported Characteristics of health care Institutions Nearby

\begin{tabular}{lll}
\hline Variables & Mean/Frequency & Range/Proportion \\
\hline Cold cost in PHC(CNY) & 152.21 & $0-3500$ \\
& & \\
Distance from home to the nearest PHC(km) & 2.83 & $0-60$ \\
Time from home to the nearest PHC(min) & 19.65 & $0-120$ \\
Distance from home to the nearest TH(km) & 23.00 & $0-400$ \\
Time from home to the nearest TH(min) & 34.56 & $0-480$ \\
Waiting time in PHC(min) & 17.81 & $0-240$ \\
Waiting time in TH(min) & 40.80 & $0-300$ \\
& & \\
Transportation fee to PHC(CNY) & 10.57 & $0-300$ \\
Number of hospitals available & 37 & \\
0 & 322 & $2.65 \%$ \\
1 & 634 & $45.42 \%$ \\
2 & 403 & $28.87 \%$ \\
$\geq 3$ & 491 & $35.17 \%$ \\
Type of nearest hospital & 63 & $4.51 \%$ \\
General hospital & 231 & $16.55 \%$ \\
Special hospital & 354 & $25.36 \%$ \\
Clinic & 148 & $10.60 \%$ \\
Community health service centre (station) & $7.09 \%$ \\
Township health centre & 99 & $35.17 \%$ \\
$\quad$ Village clinic & 10 & \\
Other &
\end{tabular}

We present the results of the logit regression analysis of factors associated with bypass behaviour for the overall population in Table 3. The results showed that referring to men, women had a relatively high odds ratio of 1.71 for bypass behaviour in the past year $(\mathrm{p}<0.01)$. The odds ratio of bypass behaviour among urban residents was 1.81 referring to that of rural residents. The farther the respondent's residence was from the PHC, the higher the odds ratio of bypass behaviour was, while the longer it took to travel to a higherlevel health care institution, the lower the odds of bypass behaviour among respondents were. In addition, as age increased, the odds of respondents' bypass behaviour tended to increase. For per increase of patients' PCAT scores, the odds ratio of for the bypass behaviour in the past 12 months was $0.98(\mathrm{p}<0.05)$.

Table 3. Regression Analysis of Factors Associated with Bypass Behaviour 


\begin{tabular}{|c|c|c|c|}
\hline \multirow{2}{*}{$\begin{array}{l}\text { Independent Variables } \\
\text { PCAT }\end{array}$} & Odds Ratio & \multicolumn{2}{|c|}{ 95\% Conf. Interval } \\
\hline & $0.98 *$ & 0.95 & 0.99 \\
\hline Female (Ref: Male) & $1.71 * * *$ & 1.27 & 2.31 \\
\hline Age & 1.02 & 0.99 & 1.04 \\
\hline Hospitalized (Ref: Outpatient) & 1.24 & 0.90 & 1.71 \\
\hline Urban (Ref: Rural) & $1.81^{* *}$ & 1.18 & 2.78 \\
\hline \multicolumn{4}{|l|}{ Marriage (Ref: Single) } \\
\hline Married & 1.90 & 0.83 & 4.38 \\
\hline Other (divorced or separated) & 3.29 & 0.83 & 13.08 \\
\hline \multicolumn{4}{|l|}{ Number of kids (Ref: 0) } \\
\hline 1 & 0.45 & 0.19 & 1.07 \\
\hline 2 & 0.52 & 0.20 & 1.31 \\
\hline 3 & 0.64 & 0.18 & 2.25 \\
\hline$>3$ & 0.67 & 0.16 & 2.79 \\
\hline \multicolumn{4}{|c|}{ Education (Ref: Elementary school or lower) } \\
\hline Junior high school & 1.11 & 0.58 & 2.13 \\
\hline High school & 1.37 & 0.68 & 2.74 \\
\hline Junior college & 0.99 & 0.46 & 2.17 \\
\hline Undergraduate & 1.26 & 0.57 & 2.77 \\
\hline Graduate student or higher & 1.27 & 0.39 & 4.09 \\
\hline \multicolumn{4}{|l|}{ Income (Ref: <5529) } \\
\hline $5529-12,899$ & 0.78 & 0.49 & 1.24 \\
\hline $12,899-20,924$ & 0.63 & 0.37 & 1.08 \\
\hline 20,924-31,990 & 1.25 & 0.67 & 2.34 \\
\hline $31,990-59,259$ & 0.85 & 0.49 & 1.44 \\
\hline$>59,259$ & 0.75 & 0.44 & 1.27 \\
\hline \multicolumn{4}{|l|}{ Occupation (Ref: <5529) } \\
\hline Teacher & 1.34 & 0.60 & 2.99 \\
\hline Public servant & 0.74 & 0.29 & 1.89 \\
\hline Worker & 0.90 & 0.48 & 1.70 \\
\hline Individual household & 0.91 & 0.48 & 1.74 \\
\hline Farmer & 0.64 & 0.28 & 1.47 \\
\hline Retired & 0.72 & 0.26 & 1.99 \\
\hline Temporary unemployment & 0.99 & 0.23 & 4.33 \\
\hline Long-term unemployment & 0.91 & 0.28 & 3.00 \\
\hline Medical staff & 0.87 & 0.45 & 1.68 \\
\hline Others & 0.86 & 0.22 & 3.43 \\
\hline \multicolumn{4}{|l|}{ Insurance (Ref: none) } \\
\hline UEBMI & 1.97 & 0.93 & 4.17 \\
\hline URBMI & 1.42 & 0.69 & 2.90 \\
\hline NCMS & 1.29 & 0.63 & 2.66 \\
\hline UREBMI & 1.93 & 0.72 & 5.16 \\
\hline Resident medical insurance & 2.03 & 0.70 & 5.89 \\
\hline Business insurance (Ref: None) & 1.02 & 0.73 & 1.43 \\
\hline Self-health grade (Ref: Very poor) & \multicolumn{3}{|c|}{ multicollinearity } \\
\hline Poor & \multicolumn{3}{|c|}{-} \\
\hline Fair & \multicolumn{3}{|l|}{-} \\
\hline Good & \multicolumn{3}{|l|}{-} \\
\hline Very good & \multicolumn{3}{|l|}{-} \\
\hline Chronic disease (Ref: None) & 1.28 & 0.81 & 2.01 \\
\hline Number of hospitals nearby & 1.13 & 0.93 & 1.37 \\
\hline Cold fee for PHC & $1.00^{*}$ & 1.00 & 1.00 \\
\hline Distance to PHC & $1.09 * *$ & 1.03 & 1.16 \\
\hline Time to PHC & 1.00 & 0.99 & 1.01 \\
\hline Distance to $\mathrm{TH}$ & 1.00 & 0.99 & 1.01 \\
\hline Time to TH & $1.00 *$ & 0.99 & 1.00 \\
\hline Wait time for PHC & 1.00 & 0.99 & 1.01 \\
\hline
\end{tabular}




\begin{tabular}{llll} 
Wait time for TH & 1.00 & 0.99 & 1.00 \\
Fare to PHC & 1.00 & 0.99 & 1.01 \\
cons & 2.34 & 0.31 & 17.62 \\
\hline
\end{tabular}

$* \mathrm{p}<0.05 \square^{* *} \mathrm{p}<0.01 \square * * * \mathrm{p}<0.001$

\section{Discussion}

This study analysed the relationship between the bypass behaviour of Chinese residents and their perception of primary health care services. The results showed that the residents' with more positive perceptions of the quality of services in PHClsperformed less bypass behaviour. Additionally, the influence of gender, household registration, distance from PHC and time to tertiary hospitals on bypass behaviour cannot be ignored. Overall, our findings demonstrated the value of improving perceptions of the quality of primary health care services to forming the patients' concept of utilizing PHC under the current loose rules of first diagnosis. This study provides a new perspective for the investigation of patients' bypass behaviour in the context of China's NHMS, which also supports the following discussion.

Our results showed that when patients perceived that primary care institutions were of higher quality, their unreasonable bypass behaviour was reduced; , thus, the improvement of perceived service quality is very important for reducing bypass behaviour and even the development of the NHMS. In China, the PHCls, which undertake the diagnosis and treatment of chronic diseases and frequently occurring diseases, effectively ensures people's health and maintains the orderly operation of the health care system, is a core component of the NHMS. However, due to the lagged development of PHC system (29), Chinese residents formed prejudice on the service quality of $\mathrm{PHCls}$, despite of the development of $\mathrm{PHC}$ system in the past decade, and tend to go to higher-level hospitals when they have health problems, resulting in unreasonable bypass behaviour. This may be because most patients perceive that $\mathrm{PHCls}$ cannot provide health care services to treat their diseases or because their previous experiences of diagnosis and treatment in primary care institutions were dissatisfactory. Previous studies have shown that better service quality in primary care institutions can allow patients to have a more comfortable experience, enhance patients' trust in PHCls, and help patients perceive a high quality of health care services, similar to those provided by secondary and tertiary hospitals. In turn, they change their concept and preference for medical care-seeking. Ultimately, the patient's tendency to go to secondary and tertiary hospitals is transferred to the preference of PHCls. Therefore, we suggest that PHCls strive to improve the patients' perceptions on quality of the services they provide, including the following elements: perfecting patients' experience of their first contact; ensuring continuous health care; respecting patients; communicating effectively with patients; and focusing on patients, families and communities(22, 23, 30-32).

In addition, household registration, health insurance, and income have impact on the patient's bypass behaviour.

In China, Medical insurance do actually have greatly affect residents' bypass behaviour. The results of this study indicate that the odds ratio of bypass behaviour for UEBMI participants referring to participants with no social insurance were the highest among participants with social health insurance, followed by 
that of URBMI participants, and the odds ratio of bypass behaviour for NCMS participants was the lowest. It is possible that medical insurance has an endogenous relationship with urban and rural household registration. The odds ratio of bypass for urban residents referring to rural residents was relatively high because in China, the types of insurance for urban residents are UEBMI and URBMI, while rural residents are generally involved in the NCMS. This finding may be explained by the following factors. First, the proportion of reimbursement for each type of medical insurance of bypass behaviour is different. In general, the lower the reimbursement rate of medical insurance is when patients bypass, the lower the probability of bypass behaviour, which must exclude patients who are not sensitive to price factors. On the other hand, people with UEBMI and URBMI living in cities have more choices for hospitals, and as our research shows, they have at least two hospitals to choose from. Therefore, they will choose a tertiary hospital with more advanced medical technology within their capabilities.

Another factor worthy of discussing is income. Although our regression results show that the impact of income on bypass behaviour is not significant, in reality, income is an important factor affecting bypass behaviour. Previous studies have shown that those with higher income have a higher odds of bypass behaviour(13). This can be explained by the fact that people with higher incomes have more social capital and are more likely to choose doctors they are familiar with when seeking medical treatment and thus bypass primary care facilities. When their social capital is situated in the primary hospital, it reduces bypass behaviour. This uncertainty also explains why our regression results are not significant. In addition, higher-income groups may also purchase commercial medical insurance, which may only be reimbursed in secondary and tertiary hospitals or other private hospitals, thus making it unnecessary for these patients to go to $\mathrm{PHCls}$.

In summary, this study suggests that the perceived quality of PHCls may play a more important role than other factors in reducing bypass behaviour. We also need further research to confirm the mechanism for patients' perceptions of quality reducing bypass behaviour. If patients' perceptions contribute to the more efficient utilization of primary health care services, the question is whether and how to manipulate these perceptions to improve the use of primary health care services, which requires further research.

\section{Limitations}

This is the first empirical study of the relationship between patient-perceived PHC quality and bypass behaviour in China. Although our results are the best approximation of the relationships investigated, some limitations must be considered.

The study did not consider the impact of provincial-level factors, including the regional culture and the policies of the provinces. For example, in the context of China's NHMS, health policies vary among different provinces, which may contribute to differences in residents' bypass behaviour. One strength of this study is that the survey data were collected nationwide, but the variables available for analysis were 
limited, because in order to retain a relatively large sample and to control the quality of the data, our survey did not cover all potential control variables. We only included significant variables based on presurvey results, which may ignore the effects of potential factors. Also, some variables were based on the respondents' memory of the last 12 months, they may have potential recall bias, such as number of annual bypass behaviours. In addition, the study did not consider the stock characteristics of PHC quality perceptions. We did not measure the relationship between PHC quality perceptions and bypass behaviour over a period of time, so there might be bias caused by time-related factors. We were unable to determine the causality between bypass behaviour and perceived quality of primary health care services over time.

\section{Conclusions}

This study suggests that patients' perceptions of the quality of primary health care services may play an active role in reducing patient bypass behaviour. Chinese residents had the highest score for family centeredness on the PCAT scale and the lowest score for community orientation. As our research shows, the quality perception of primary care institutions plays a major role in patient bypass behaviour and may produce significant spillover effects in China. We recommend that government departments improve and invest in the quality of PHCls and focus on rectification in areas with low PCAT scores to improve patients' trust in and preferences for $\mathrm{PHCls}$ by improving their visit experiences.

\section{List Of Abbreviations}

NHMS= national hierarchical medical system

$\mathrm{PHC}=$ primary health care

\section{Declarations}

\section{Ethics approval and consent to participate}

The ethical approval to conduct the pilot survey and main survey was granted by the Ethics Committee of China Pharmaceutical University (Project Number: CPU2019015).

Written consent to participate was obtained from each participant before data collecting.

\section{Consent for publication}

No sensitive and personal data were recorded, while confidentiality of data was assured during data analysis and reporting.

\section{Availability of data and materials}


The datasets used and/or analysed during the current study are available from the corresponding author on reasonable request.

\section{Competing interests}

The authors declare that they have no competing interests

\section{Funding}

None.

\section{Authors' contributions}

WZ, YH and XX contributed to conception and design of the work; WZ, GL and HZ contributed to the acquisition and analysis of the data; $\mathrm{WZ}, \mathrm{YH}$ and $\mathrm{XX}$ contributed to the interpretation of data; $\mathrm{WZ}, \mathrm{YH}$ and GL contributed to drafting of the work; WZ and YH contributed to revision of the work. All authors read and approved the final manuscript.

\section{Acknowledgements}

The authors thank all investigators recruited for data collection and all participants of the survey.

\section{References}

1. Hall JJ, Taylor R. Health for all beyond 2000: the demise of the Alma-Ata Declaration and primary health care in developing countries. 2003;178(1):17-20.

2. Walt G, Vaughan P. An introduction to the primary health care approach in developing countries. A review with selected annotated references. 1981.

3. Li F. Analysis on the specific path and practice degree of medical service classification diagnosis and treatment in China. Academic Journal of Zhongzhou. 2014(11):90-5.

4. Reinhardt UE, Cheng TM. The world health report 2000 - Health systems: improving performance. Bulletin of the World Health Organization. 2000;xvi(8):1064.

5. Aoki T, Yamamoto Y, Ikenoue T, Kaneko M, Kise M, Fujinuma Y, et al. Effect of Patient Experience on Bypassing a Primary Care Gatekeeper: a Multicenter Prospective Cohort Study in Japan. Journal of general internal medicine. 2018;33(5):722-8.

6. Rieber GM, Benzie D, McMahon S. Why patients bypass rural health care centers. Minnesota medicine. 1996;79(6):46-50. 
7. Radcliff TA, Brasure M, Moscovice IS, Stensland JT. Understanding rural hospital bypass behavior. J Rural Health. 2003;19(3):252-9.

8. Gauthier B, Wane W. Bypassing health providers: the quest for better price and quality of health care in Chad. Social science \& medicine. 2011;73(4):540-9.

9. Akin JS, Hutchinson P. Health-care facility choice and the phenomenon of bypassing. Health Policy Plann. 1999;14(2):135-51.

10. China NHCotPsRo. 2017 Statistical Bulletin on the Development of China's Health Care. 2018.

11. Leonard KL, Mliga GR, Mariam DH. Bypassing health centers in Tanzania: Revealed preferences for observable and unobservable quality. 2002;11(102-43):947-60(14).

12. Radcliff TA, Brasure M, Moscovice IS, Stensland JT. Understanding Rural Hospital Bypass Behavior. Journal of Rural Health. 2003;19(3):252-9.

13. Rao KD, Sheffel A. Quality of clinical care and bypassing of primary health centers in India. Social science \& medicine. 2018;207:80-8.

14. Kahabuka C, Moland KM, Kvale G, Hinderaker SG. Unfulfilled expectations to services offered at primary health care facilities: Experiences of caretakers of underfive children in rural Tanzania. Bmc Health Services Research. 2012;12.

15. Damrongplasit K, Wangdi T. Healthcare utilization, bypass, and multiple visits: the case of Bhutan. International journal of health economics and management. 2017;17(1):51-81.

16. Kruk ME, Mbaruku G, Mccord CW, Moran M, Rockers PC, Galea S. Bypassing primary care facilities for childbirth: a population-based study in rural Tanzania. 2009;24(4):279-88.

17. Du X, Ling C. Analysis of Influencing Factors of Guangxi Residents' Medical Treatment Flow. Statistics and Management. 2018;253(08):99-102.

18. Ning M. Research on the Impact of New Rural Cooperative Medical Care Partial Burden System on Farmers' Hospital Choice Behavior. Journal of Agrotechnical Economics. 2014(1):113-21.

19. Yna J, Lan Y. Incomplete Information and Disordered Self-Selection--A Theoretical Explanation on the Failure of Reasonable Patients' Distribution. Zhejiang Academic Journal. 2019;235(02):2+18796.

20. Yao Z. Health reform dilemma: disorderly medical freedom after government retreat. Beijing Cultural Review. 2015(05):49-56.

21. Zhu X, Yang G, Chen M, Song P, Chen X. Study on the Influencing Factors of Health-seeking Behaviors in Patients with Chronic Diseases. Journal of Chengdu Medical College. 2019;14(02):119-23.

22. Han Z. Study on Health Seeking Behavior and Satisfaction of Inpatients in Rural Hospital Based on Medical Service Distribution: Shandong University; 2012.

23. Kang X. Analysis on the Influencing Factors of Hospital Selecting Behavior in Wuhan: Central China Normal University; 2016.

24. Tang S. The Research on Medical Behavior and Impact Factors of Urban Residents: Southwestern University of Finance and Economics; 2014. 
25. China NHcotPsRo. Basic situation of development of primary health care system. In: department Ph, editor.

2020.http://www.nhc.gov.cn/jws/s3578/202002/59310c05e27944dabfe68bccc405561d.shtml.

26. Shi L, Starfield B, Xu J, Politzer R, Regan J. Primary care quality: community health center and health maintenance organization. 2003;96(8):787.

27. Yang H, Shi LY, Lebrun LA, Zhou XF, Liu JY, Wang H. Development of the Chinese primary care assessment tool: data quality and measurement properties. Int J Qual Health C. 2013;25(1):92-105.

28. Dullie L, Meland E, Mildestvedt T, Hetlevik $\emptyset$, Gjesdal S. Quality of primary care from patients' perspective: a cross sectional study of outpatients' experience in public health facilities in rural Malawi. BMC Health Services Research. 2018;18(1).

29. Li X, Lu JP, Hu S, Cheng KK, De Maeseneer J, Meng QY, et al. The primary health-care system in China. Lancet. 2017;390(10112):2584-94.

30. Lu Y. Analysis on the influencing factors of the health seeking behavior of the elderly in China. China Journal of Pharmaceutical Economics. 2017(4).

31. Lu B. Research on Residents Health Seeking Behavior in Gansu Province: Lanzhou University; 2012.

32. Zhang L, Li W. Investigation on the Influencing Factors of the Medical Behaviors of Urban and Rural Residents in Shandong Province. Medicine \& Philosophy(A). 2016(23). 